\title{
Simultaneous Differential Scanning Calorimetry-Synchrotron X-ray Powder Diffraction: A Powerful Technique for Physical Form Characterization in Pharmaceutical Materials
}

\author{
Alexander Clout, ${ }^{\dagger}$ Asma B. M. Buanz, ${ }^{\dagger}$ Timothy J. Prior, ${ }^{\ddagger}$ Christina Reinhard, ${ }^{\S}$ Yue Wu, \\ Dermot O'Hare, "Gareth R. Williams, ${ }^{* \dagger}$ and Simon Gaisford ${ }^{* \dagger}$ \\ ${ }^{\dagger}$ UCL School of Pharmacy, University College London, 29-39 Brunswick Square, London, WC1N 1AX, United Kingdom \\ ${ }^{\ddagger}$ Department of Chemistry, University of Hull, Cottingham Road, Hull, HU6 7RX, United Kingdom \\ ${ }^{\S}$ Diamond Light Source, Harwell Science and Innovation Campus, Didcot, Oxfordshire, OX11 0DE, United Kingdom \\ "Chemistry Research Laboratory, Department of Chemistry, University of Oxford, 12 Mansfield Road, Oxford, OX1 3TA, United \\ Kingdom
}

\section{Supporting Information}

ABSTRACT: We report a powerful new technique: hyphenating synchrotron X-ray powder diffraction (XRD) with differential scanning calorimetry (DSC). This is achieved with a simple modification to a standard laboratory DSC instrument, in contrast to previous reports which have involved extensive and complex modifications to a DSC to mount it in the synchrotron beam. The high-energy X-rays of the synchrotron permit the recording of powder diffraction patterns in as little as $2 \mathrm{~s}$, meaning that thermally induced phase changes can be accurately quantified and additional insight on the nature of phase transitions obtained. Such
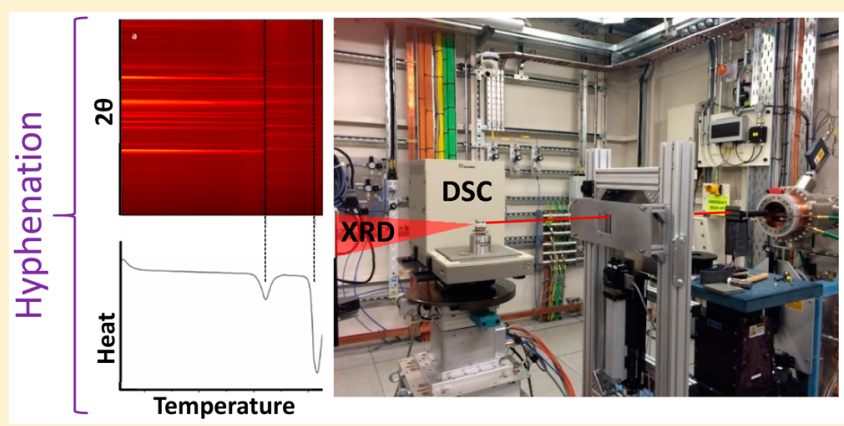
detailed knowledge cannot be gained from existing laboratory XRD instruments, since much longer collection times are required. We demonstrate the power of our approach with two model systems, glutaric acid and sulfathiazole, both of which show enantiotropic polymorphism. The phase transformations between the low and high temperature polymorphs are revealed to be direct solid-solid processes, and sequential refinement against the diffraction patterns obtained permits phase fractions at each temperature to be calculated and unit cell parameters to be accurately quantified as a function of temperature. The combination of XRD and DSC has further allowed us to identify mixtures of phases which appeared phase-pure by DSC.

$\mathrm{D}$ ifferential scanning calorimetry (DSC) is an extremely widely used tool for studying temperature-driven phase changes in solid materials. Its utility stems from the fact that nearly all phase changes are accompanied by a change in heat content and so it can be applied to virtually any sample. Heat transfer, however, is a ubiquitous parameter that does not provide molecular or structural insight into the source from which it originated. Further, other events such as decomposition, sintering, separation, foaming and bubbling, and creep of melts may give rise to heat changes that are not actually a result of phase transitions in the sample. Thus, while DSC analysis in isolation can be extremely informative, absolute assignment of the changes in physical form which occur with each phase transition is impossible without ancillary data.

Two common experimental approaches to interpret thermal data and to identify and assign phase changes are to change the DSC operating conditions or to analyze the sample with thermogravimetric analysis (TGA). Changing the DSC heating rate determines whether phase transitions are thermodynamic (e.g., melting) or kinetic (e.g., recrystallization) in nature, because thermodynamic transitions do not show a dependence on heating rate while kinetic transitions do. TGA allows determination of whether the sample loses volatile components or degrades with temperature. However, while both approaches aid interpretation, neither affirms what actual structural changes in phase have occurred.

It is of course possible to take sample material directly from the DSC and analyze it with another technique, but this approach can only be used to identify the material at the measurement temperature and the assumption must be made that the sample does not change between removal from the DSC and subsequent analysis (potentially an issue for metastable or intermediate phases). The best option therefore is in situ analysis of the sample while it is being heated in the DSC itself, by linking a second instrument to the calorimeter

Received: July 5, 2016

Accepted: September 19, 2016

Published: September 19, 2016 
(so-called hyphenated techniques). ${ }^{1}$ Such an approach ensures that the temperature of the sample is known and controlled throughout the measurement and, if the secondary method of analysis is sufficiently rapid, information on physical form can be collected in real time for all the phases the material may progress through. The additional analysis should ideally be noninvasive, so as not to affect the temperature of the sample or to introduce further erroneous heats, and hence spectroscopic probes are often used. For instance, DSC has been hyphenated with Fourier transform-infrared (FT-IR), ${ }^{2}$ nearinfrared (NIR), ${ }^{3}$ and Raman $^{4-8}$ spectrometers and light microscopy (to make simple optical observations). ${ }^{9}$

While these approaches have proved extremely powerful, the "gold-standard" analytical tool for the characterization of physical form remains X-ray diffraction (XRD). XRD analysis can provide absolute determination of the position of atoms and molecules within unit cells, and laboratory variabletemperature XRD has led to useful insights in the changes occurring to the structure of a sample upon heating. ${ }^{10,11}$ Unfortunately, standard equipment is not able to deliver the high heating rates and short data collection times used in DSC, and thus its application to in situ DSC analysis is limited. However, the data collection time can be reduced significantly if a synchrotron X-ray source (SX) is used. Researchers have for around 20 years employed SX to explore the mechanisms and kinetics of solid-state reactions, initially using powerful white beams of X-rays to penetrate bulky reaction vessels. ${ }^{12}$ This energy-dispersive technique suffered from poor spatial resolution, making high-quality structural information difficult to obtain. Recent developments in both synchrotron and detector technologies allow the combination of high-energy monochromatic X-rays and exquisitely sensitive detectors to obtain high-resolution diffraction patterns on samples in as little as $1 \mathrm{~s}$ or less. ${ }^{13}$ Hence, it is now feasible to obtain high-quality data suitable for structural (Rietveld) refinement from the direct hyphenation of SX and DSC.

Several research groups have mounted DSC instruments in synchrotron X-ray sources. For instance, Gilbert et al. ${ }^{14}$ studied the influence of quenching on the phase separation of unstable $n$-alkane blends with DSC-wide-angle X-ray scattering. Using the same technique, Bayés-Garcia et al. ${ }^{15}$ studied transformation pathways of 1,3-dipalmitoyl-2-oleoyl glycerol and Brubach et al. ${ }^{16}$ explored transitions in glyceryl behenate. All three groups used modified DSC pans to permit transmission of the synchrotron $\mathrm{X}$-ray beam, generally adding mica windows to the pans or holding the sample in glass capillaries. These modifications have the potential to alter the pathways of heattransfer from the sample to the DSC and so to affect the DSC data. The apparatus required is also complex and the experiment difficult to implement in comparison to a simple lab DSC.

Although these effects might be small, we wondered whether it was possible to construct an experiment to use unmodified DSC pans in a synchrotron source and so minimize errors in DSC measurement and maximize the ease by which lab DSC protocols can be translated into DSC-XRD. The DSC 2010 series of heat-flux DSC instruments manufactured by TA Instruments LLC have the advantage that the element used to heat the sample and reference pans is coiled around the cell (see Figure 1). The heat generated is transferred to the DSC head via a metal shield ( $\sim 5 \mathrm{~mm}$ thickness). Ordinarily this shield would be impenetrable to a synchrotron X-ray beam, but it is possible to drill holes through it to allow the beam to pass

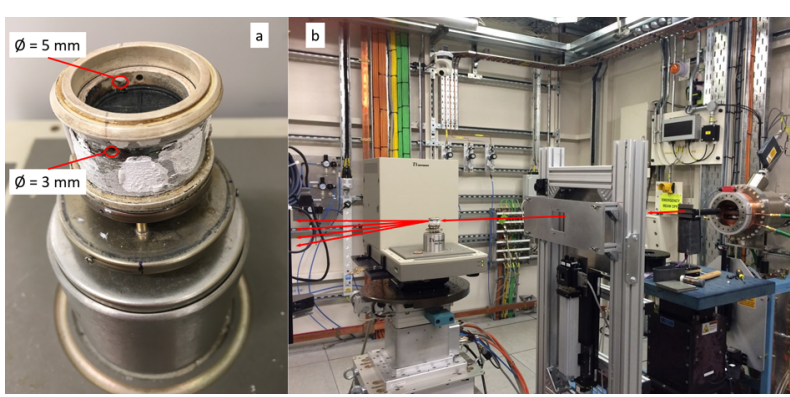

Figure 1. Photographs of (a) the modified DSC head and (b) the DSC positioned on Beamline I12 at the Diamond Light Source. The red line denotes the path of the SX beam.

unhindered. Correct placing of the holes means that the sample pan can be located in the direct path of the synchrotron X-ray beam, with the reference pan to one side. Further, the pans themselves are located on raised bases (which contain the thermocouples), which means that the diffracted X-ray beam can exit the instrument without contacting any part of it (Figure 1). The pans are made of thin aluminum, through which the synchrotron beam can pass. Although X-rays are scattered by aluminum, this gives only a few Bragg reflections which can easily be modeled or excluded from analysis. With this arrangement, DSC data and diffraction patterns can be collected simultaneously on a single sample, using a standard laboratory calorimeter.

Here we report the modifications to the DSC required to allow the use of unmodified pans and apply the method to the analysis of two model compounds, both of which have enantiotropically related polymorphs: glutaric acid and sulfathiazole.

\section{MATERIALS AND METHODS}

Simultaneous differential scanning calorimetry-synchrotron Xray powder diffraction (DSC-XRD) experiments were carried out on the Joint Engineering Environmental and Processing beamline I12 (JEEP) at the Diamond Light Source. ${ }^{17}$ The beamline produces a continuous spectrum of X-rays in the energy range $50-150 \mathrm{keV}$, which for these experiments was monochromated to $53.16 \mathrm{keV}$. The diameter of the beam was $0.5 \mathrm{~mm}$. A Thales Pixium RF4343 detector was positioned 1.9 $\mathrm{m}$ behind the sample. Detector calibration was performed using cerium dioxide. Diffraction patterns were recorded every few seconds (data were collected for $2 \mathrm{~s}$ with a pause between collections).

DSC measurements were performed with a TA 2010 instrument (TA Instruments LLC). The DSC furnace was modified by drilling a hole either side of the sample holder to allow unhindered passage of the X-ray beam through the sample. Prior to drilling, the heating coils were carefully separated such that they were placed either side of the holes. Heat sink paste was applied post-drilling to insulate against short circuiting of the instrument. The entry hole was $3 \mathrm{~mm}$ in diameter and the exit hole $5 \mathrm{~mm}$ (to account for diffraction of the X-ray beam), as shown in Figure 1a. Calibration was performed with a certified indium standard according to the manufacturer's instructions. Samples $(\sim 30 \mathrm{mg})$ of glutaric acid or sulfathiazole were held in Tzero aluminum pans and heated at $10{ }^{\circ} \mathrm{C} \mathrm{min}{ }^{-1}$ from 30 to $180{ }^{\circ} \mathrm{C}$ and $220{ }^{\circ} \mathrm{C}$, respectively. It was found that the $\mathrm{X}$-ray beam was sufficiently intense to pass through the thin aluminum pans, and so no modification of the 
pans was necessary. DSC data were recorded with the TA Advantage software package, analyzed with TA Universal Analysis, and plotted with Origin 9.1.

XRD data were analyzed as follows. The large grain size of the samples resulted in unrepresentative spots of high intensity in the 2D Pixium data, and thus the DAWN Science Workbench was first used to mask these regions of the 2D data. The same software was used to convert the $2 \mathrm{D}$ data into 1D diffraction patterns. ${ }^{18}$ Contour plots of the raw XRD data were then plotted using bespoke software routines. Selected patterns were first analyzed by the Rietveld method implemented within GSAS, ${ }^{19}$ in order to obtain realistic values for the unit cell parameters at elevated temperatures. Backgrounds were fitted using a 6-term shifted Chebychev polynomial of the first kind. Lattice parameters and a single Gaussian peak shape parameter were refined. In cases where more than one phase was present, the peak shapes for each phase were constrained to be the same and the phase fraction refined. The models used came from the CCDC (details are given below). The atom positions were not refined. Atom displacement parameters, $U_{\text {iso }}$ were set to be $0.15 \AA^{2}$ in each phase.

The TOPAS-Academic suite of programs ${ }^{20}$ was further employed to perform batch refinements of all data sets collected, using the same procedures as in GSAS. No zero point was refined as entire diffraction patterns were collected using a $2 \mathrm{D}$ area detector.

\section{RESULTS AND DISCUSSION}

Apparatus. Before analyzing any samples it was necessary to assess the effect of drilling holes in the DSC furnace. Calibration was carried out with a certified reference sample of indium and a clear single melt was observed with onset and enthalpy values within the manufacturer's specified tolerances. Temperature versus time data for the modified DSC 2010 were recorded and compared with the same heating profile on an unmodified DSC, and the results can be seen in Figure 2. It is

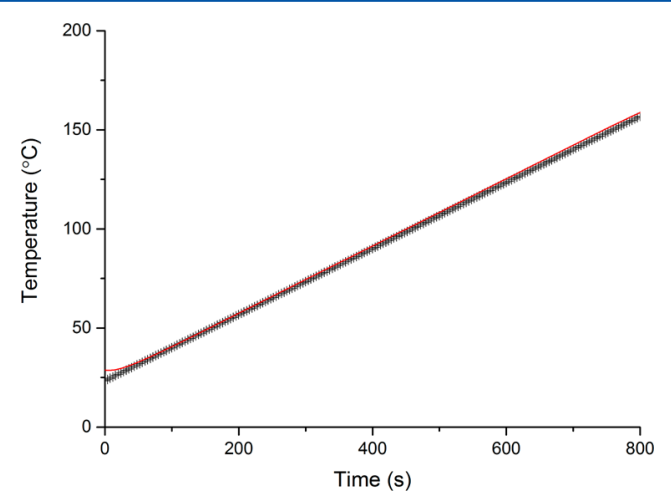

Figure 2. Measured temperature versus time data for the modified DSC 2010 (red line) and an unmodified Q20 DSC (black crosses).

clear from the linear nature of the heating rate and its similarity to that of the unmodified DSC that the ability of the DSC 2010 to control temperature and heat was not compromised by the addition of the holes.

Sulfathiazole. To aid interpretation of the data, the XRD patterns were first converted to contour plots; these show peak intensities as a color-scale as a function of temperature and permit easy visualization of changes in crystalline form. DSCXRD data for sulfathiazole are given in Figure 3. The DSC data
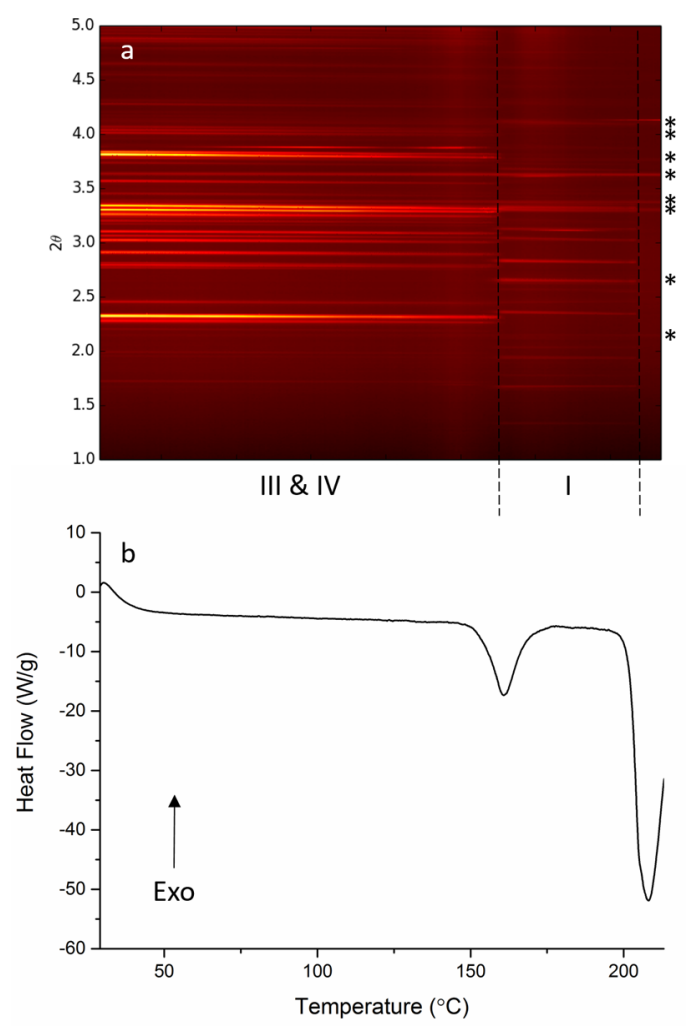

Figure 3. DSC-XRD data for sulfathiazole. (a) A contour plot of the diffraction data and (b) the corresponding DSC trace. Some residual reflections, marked $*$, persist above $202{ }^{\circ} \mathrm{C}$; these arise from diffraction by the furnace elements.

show two clear endotherms with onsets at 154 and $202{ }^{\circ} \mathrm{C}$. The former corresponds to a stark change in the diffraction pattern, while the latter is coincident with the complete disappearance of all diffracted intensity from the sample and agrees well with the reported melting point of sulfathiazole form I at $201{ }^{\circ} \mathrm{C}$. ${ }^{21}$ It is important to note that there are no unexpected events in the DSC data, and that the observed transitions all occur at the temperatures expected from the literature. We have calculated the X-ray absorption cross-section of the samples, and even on the most pessimistic assumptions find heating from the X-ray beam to be $<0.1{ }^{\circ} \mathrm{C} \mathrm{min}^{-1}$. Hence, while a small amount of local heating of the sample by the beam may occur, the DSC is able to effectively compensate for this and the heating profile is unaffected by the exposure to X-rays.

The changes in the diffraction pattern are complete at the apex of the first endotherm in the DSC trace, indicating the phase transition is complete here and the return of the heat flow to baseline can be attributed to the instrument and not the sample. Form III is reported to be the most stable form of sulfathiazole between 10 and $100{ }^{\circ} \mathrm{C},{ }^{21}$ and the temperature of the first endotherm corresponds well to literature values for a solid-solid phase transition from form III to I, reported to occur between 150 and $170{ }^{\circ} \mathrm{C}$. ${ }^{22}$

To verify the assignment of the phases present in each segment of the contour plot, selected patterns at 29 and $161^{\circ} \mathrm{C}$ were analyzed using the Rietveld method (Figure 4 and Table 1). A relatively poor fit was obtained at $29^{\circ} \mathrm{C}$ when considering only form III in the model (see the Supporting Information, Figure S1), and a more detailed analysis revealed that in fact the material present at this temperature was a mixture of forms III $(67.6 \%)$ and IV (32.4\%) (Figure S2). This was somewhat 

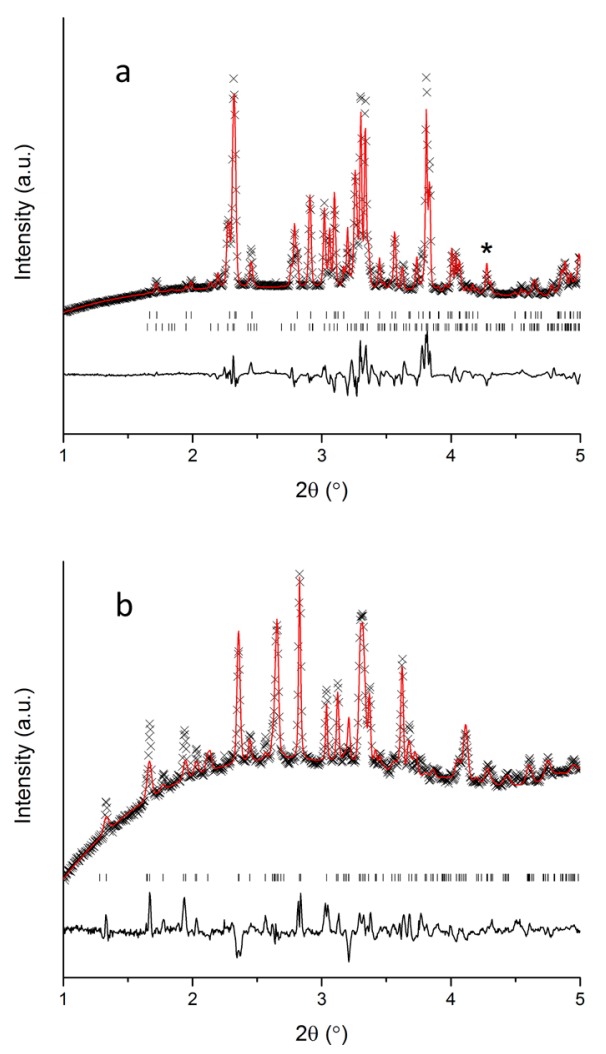

Figure 4. X-ray powder diffraction patterns for sulfathiazole at (a) 29 ${ }^{\circ} \mathrm{C}$ and (b) $161^{\circ} \mathrm{C}$. Observed (crosses), calculated (upper line), and difference (lower line) diffraction profiles are shown. The tick marks show the positions of allowed reflections from sulfathiazole form III (a, lower), form IV (a, upper), and form I (b), and the asterisk in part a denotes a characteristic reflection of form III.

Table 1. Refinement Parameters for Sulfathiazole

\begin{tabular}{|c|c|c|c|}
\hline property & form III & form IV & form I \\
\hline$T /{ }^{\circ} \mathrm{C}$ & & & 161 \\
\hline space group & $P 2_{1} / c$ & $\begin{array}{llll}P & 1 & 1 & 2 \\
1\end{array} / n$ & $P 2_{1} / c$ \\
\hline$a / \AA$ & $17.564(1)$ & $10.864(1)$ & $10.574(1)$ \\
\hline$b / \AA$ & $8.560(1)$ & $11.441(2)$ & $13.715(2)$ \\
\hline$c / \AA$ & $15.572(2)$ & $8.543(2)$ & $16.893(3)$ \\
\hline$\alpha /^{\circ}$ & 90 & 90 & 90 \\
\hline$\beta /^{\circ}$ & $112.932(9)$ & 90 & $108.596(19)$ \\
\hline$\gamma /^{\circ}$ & 90 & $91.852(12)$ & 90 \\
\hline$R_{\mathrm{wp}}$ & 0.0561 & & 0.0242 \\
\hline$R_{\mathrm{p}}$ & 0.0368 & & 0.0164 \\
\hline Phase fraction ${ }^{b}$ & 67.6 & 32.4 & 100 \\
\hline
\end{tabular}

${ }^{a}$ The starting models were taken from the CSD (form III, SUTHAZ02; form IV, SUTHAZ04; form I, SUTHAZ43). ${ }^{b}$ Because of the graininess of the sample, representative errors cannot be calculated but phase fractions for the initial mixture of sulfathiazole are reported as individually refined patterns in the Supporting Information, Figure S2.

unexpected as form III is reported to be the most stable polymorph at room temperature, ${ }^{21}$ although the stability difference between form III and IV is relatively small. ${ }^{21}$ As expected, form I was not observed to be present at this temperature. In contrast, after the transformation endotherm the diffraction pattern can be successfully fitted using the known structure for form I. It should be noted that the imperfect final fit of the patterns can be attributed to the presence of some larger crystallites in the samples, which resulted in spots of intense diffraction rather than the smooth rings expected of a finely ground powder.

The quality of the data collected permitted batch Rietveld refinement against all the patterns, giving fits with weighted profiles $\left(R_{\mathrm{wp}}\right)$ in the range $0.0200-0.0558$. The unit cell parameters could be extracted from these and plotted as a function of temperature (Supporting Information, Figures S3S5). As expected, increasing the temperature of the sample causes expansion of the unit cell in all three dimensions for all three polymorphs present, with the exception of the $c$ axis of form I which exhibited little change. To the best of our knowledge, this is the first time that the structures of purely organic species have been refined using data collected on JEEP and also the first example of refinement using data collected during a live DSC run.

It is often assumed in the literature that when phase transitions between forms are observed in DSC experiments, there is a melt of one form followed by recrystallization of a new form. ${ }^{23,24}$ The constant presence of reflections in the XRD contour plot indicates that there is no wholesale melt here, however; if the entire sample melted we would expect to see a diffraction pattern devoid of reflections. This suggests that the transition between the crystalline forms of sulfathiazole occurs via a direct solid-solid pathway, rather than by meltrecrystallization. That said, these measurements are bulk averaged over many particles and microscopic transient melting on a per particle basis cannot be ruled out.

In order to gain a more quantitative understanding of the transformation, the individual contributions of each polymorph to the overall pattern at each temperature were integrated and the results normalized to obtain a phase fraction. The results are given in Figure 5. Below $155^{\circ} \mathrm{C}$ the relative contributions

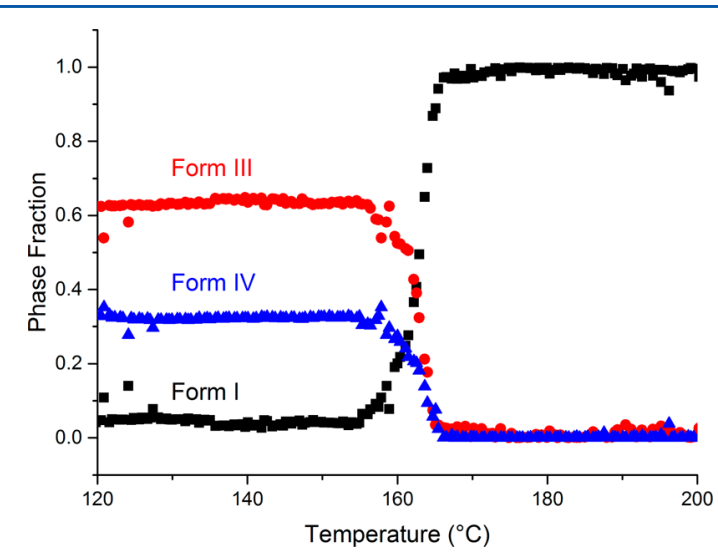

Figure 5. Plot of phase fraction vs temperature for the heat-induced transformation of sulfathiazole from a mixture of forms III (red circles) and IV (blue triangles) to form I (black squares).

of the three polymorphs are constant, with $67.6 \%$ form III and $32.4 \%$ form IV present and form I absent. Subsequently, the amount of form I begins to increase, while those of III/IV diminish simultaneously until $166{ }^{\circ} \mathrm{C}$ when the conversion is complete. If the phase fractions of forms III and IV are summed, a plot of their combined phase fraction vs temperature crosses that of form I around 0.5, indicating a solid-solid phase transition (Supporting Information, Figure S6). It is of particular interest that the transition to form I occurs simultaneously and via a solid-solid pathway for both forms III and IV. 
The transformations of forms III and IV to form I are known to occur in the temperature range $150-170{ }^{\circ} \mathrm{C} .{ }^{22}$ Form III is reported to undergo conversion via either a melt ${ }^{7}$ or a solidsolid pathway ${ }^{25}$ depending on the purity of the sample, and form IV has been shown to undergo a solid-solid transformation. ${ }^{22}$ The data collected here show that when a mixture of forms III and IV are heated both undergo a solid-solid transformation to form I simultaneously. The differences in the structure of these forms ${ }^{22}$ are very small and evidently not sufficient to make the transition from one to the other energetically more favorable than their conversion to the high temperature polymorph (see additional discussion in the Supporting Information). It is unlikely to be coincidental that the conversion happened at the same temperature for both.

Glutaric Acid. Figure 6a shows the contour plot for glutaric acid (GA) and the corresponding thermal trace recorded by the
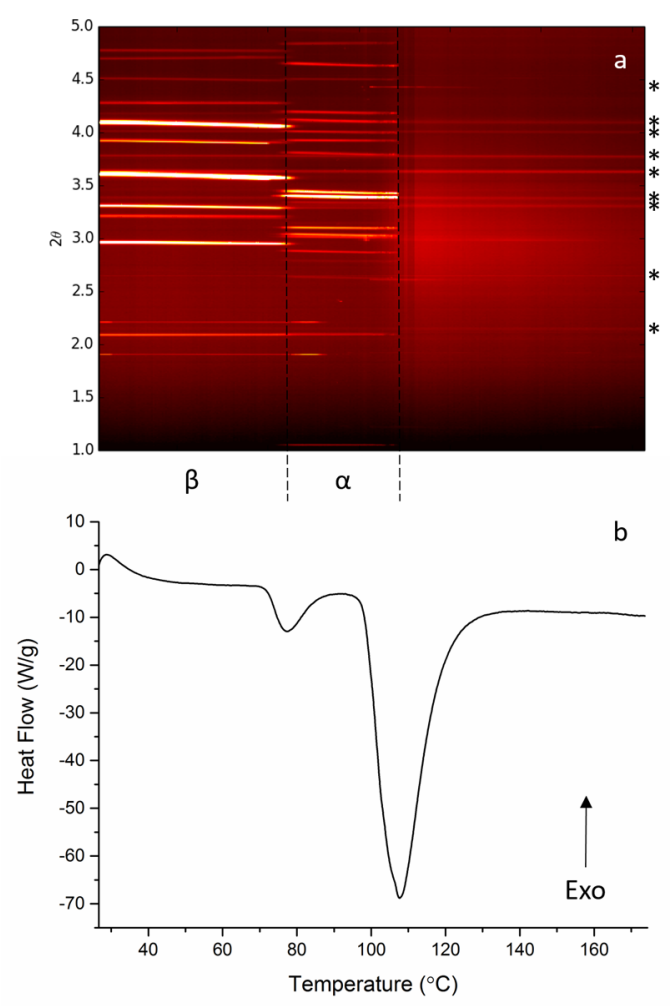

Figure 6. DSC-XRD data for glutaric acid. (a) Contour plot of the diffraction data and (b) the corresponding DSC thermogram. Some residual reflections, marked $*$, persist above $100{ }^{\circ} \mathrm{C}$; these arise from diffraction by the furnace elements.

DSC. It is immediately apparent from the contour plot that GA exists in two distinct crystalline phases, with an abrupt change in the diffraction pattern at $72{ }^{\circ} \mathrm{C}$. There is then a complete loss of diffracted intensity at $\sim 100{ }^{\circ} \mathrm{C}$. The DSC data in Figure $6 \mathrm{~b}$ contain two clear endotherms, one with an onset at $72{ }^{\circ} \mathrm{C}$ and one at $98^{\circ} \mathrm{C}$. The latter occurs at the same temperature as the loss of diffracted intensity in XRD and corresponds to the known melting point of the $\alpha$ form of GA. ${ }^{26}$ The former corresponds to the abrupt changes in diffraction pattern seen at $\sim 72{ }^{\circ} \mathrm{C}$ and thus can be ascribed to the previously reported transition between the $\beta$ (low temperature) and $\alpha$ (high temperature) forms of GA. As for sulfathiazole, the changes in the XRD contour plot are complete at the apex of the first endotherm, and the return to baseline is attributable to the instrument rather than the sample.

As for sulfathiazole, Rietveld refinements were undertaken on selected patterns. The fits are depicted in Figure 7 with
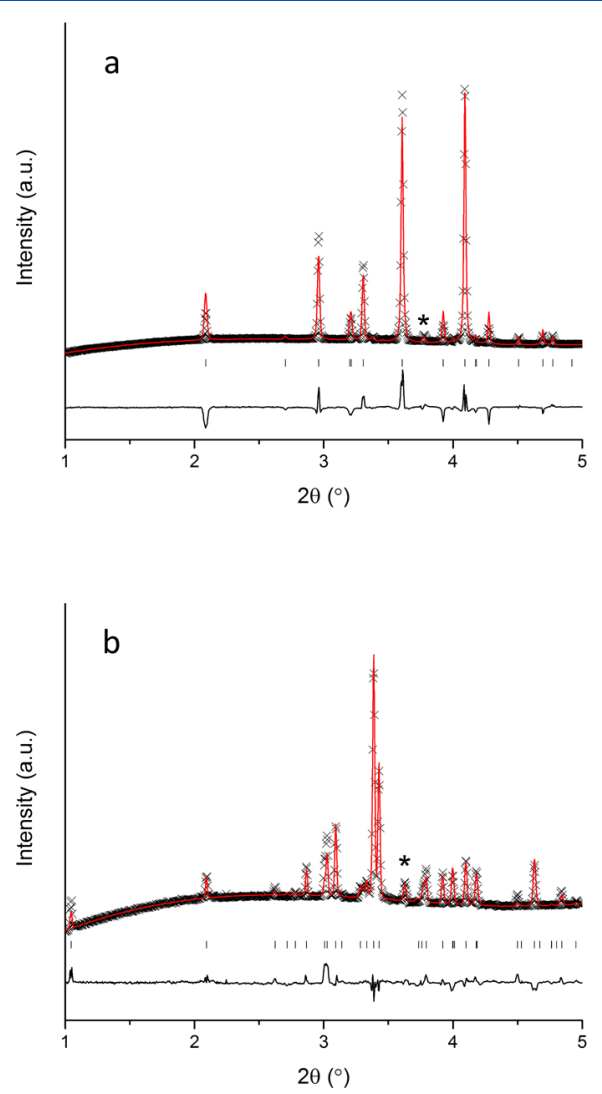

Figure 7. X-ray powder diffraction patterns for GA at (a) $27^{\circ} \mathrm{C}$ and (b) $94{ }^{\circ} \mathrm{C}$. Observed (crosses), calculated (upper line), and difference (lower line) diffraction profiles are shown. The tick marks show the positions of allowed reflections from $\beta$-GA in part a and $\alpha$-GA in part b. (The starred peak shows the presence of a persistent impurity).

refinement parameters in Table 2. The lower temperature pattern can be successfully fitted using an expanded version of the known structure for $\beta$-GA (recorded at $-153{ }^{\circ} \mathrm{C}$ ), ${ }^{27}$ and that at $94{ }^{\circ} \mathrm{C}$ similarly with the $\alpha$-form. The fit parameters are all acceptable, and it is clear that a single phase exists at each temperature.

\section{Table 2. Refinement Parameters for Glutaric Acid}

\begin{tabular}{lll}
\multicolumn{1}{c}{ property } & $\beta$-glutaric acid & $\alpha$-glutaric acid \\
$T /{ }^{\circ} \mathrm{C}$ & 27 & 94 \\
space group & $C 2 / c$ & $C 2 / c$ \\
$a / \AA$ & $12.902(1)$ & $25.577(3)$ \\
$b / \AA$ & $4.826(0)$ & $5.015(0)$ \\
$c / \AA$ & $9.960(1)$ & $10.200(2)$ \\
$\alpha /$ deg & 90 & 90 \\
$\beta /$ deg & $96.838(8)$ & $92.531(10)$ \\
$\gamma /$ deg & 90 & 90 \\
$R_{\mathrm{wp}}$ & 0.0623 & 0.0267 \\
$R_{\mathrm{p}}$ & 0.0353 & 0.0158
\end{tabular}

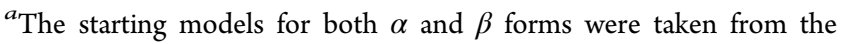
CSD (IDs: GLURAC06 and GLURAC12, respectively). 
As for sulfathiazole, batch refinements were carried out on the entire data set. Rietveld refinements yielded $R_{\text {wp }}$ values ranging from 0.0238 to 0.0621 . Because of the smaller, sidecentered, unit cells and the presence of just two polymorphs, there were fewer instances of overlapping reflections than for sulfathiazole and so, in addition to Rietveld, it was possible to carry out batch Pawley refinements (resultant $R_{\text {wp }}$ between 0.0120 and 0.0197 ). Both sets of refined data show that upon heating, rather than the unit cell expanding in three dimensions as might be expected, it expands in the $b$ and $c$ axes and $a$ and $\beta$ contract. This is true for both the $\beta$ and $\alpha$ forms of GA (Supporting Information Figures S7 and S8).

As for sulfathiazole, the contribution of each polymorph was obtained via Rietveld refinement and the phase fraction calculated. A plot of phase fraction vs temperature is given in Figure 8. As with sulfathiazole, the curves for the two forms

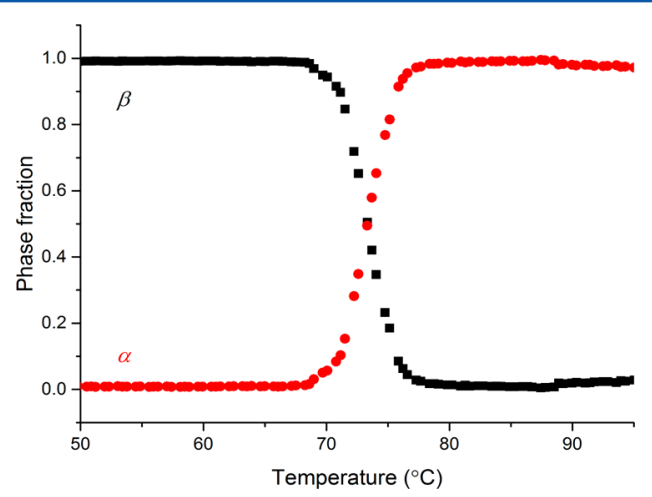

Figure 8. Plot of phase fraction vs temperature for the heat induced transformation of GA from the $\beta$ phase (black squares) to the $\alpha$ phase (red circles).

cross at $\sim 0.5$, indicating that conversion between them occurs via a solid-solid transition with no observable melt or intermediate phase. As previously though, melt-recrystallization on the microscopic scale cannot be discounted.

These observations are in agreement with the literature. Jorunn and Samuelsen ${ }^{28}$ used Raman spectroscopy to study the effect of temperature on the crystal structure of the $\beta$ polymorph, and Espeau et al. ${ }^{26}$ investigated the effects of an increase in pressure on the nature of the phase transition. Both report a solid-solid transformation from $\beta$ to $\alpha$.

\section{CONCLUSIONS}

We demonstrate in this work that hyphenated DSCsynchrotron XRD experiments can easily be performed by making only a simple modification to a laboratory DSC instrument. Using a high-intensity synchrotron X-ray source (Beamline I12, Diamond Light Source) it is possible to collect diffraction patterns on a sample mounted in a conventional DSC pan in a few seconds, permitting a single sample to be effectively interrogated both in terms of its thermal and structural features. The potency of our approach is demonstrated through a study of two systems displaying enantiotropic polymorphism. Direct, seemingly solid-solid, phase transitions are observed, with no wholesale melting of the sample. The XRD data obtained are of sufficient quality to permit Rietveld refinements to be performed and, in favorable instances, Pawley refinements. Batch refinements can be undertaken allowing a rapid $(<30 \mathrm{~min})$ analysis of the hundreds of patterns comprising each data set. This provides a detailed description of structural changes as a function of temperature. DSC-XRD thus comprises a simple and powerful analytical tool with much promise in pharmaceutical science and materials chemistry. We are currently working to make our apparatus available as a user facility, to ensure that the research community can make full use of this technique. In the longer term, given the rapid improvement in area detector technology in laboratory XRD instruments, we believe that it should be possible to produce a lab-based instrument which can obtain XRD data on similar time scales to those used in this work.

\section{ASSOCIATED CONTENT}

\section{S Supporting Information}

The Supporting Information is available free of charge on the ACS Publications website at DOI: 10.1021/acs.analchem.6b02549.

Further XRD data, including TOPAS fits for sulfathiazole, plots showing the variations in unit cell parameters with temperature for both sulfathiazole and glutaric acid, an additional phase fraction plot for sulfathiazole, and discussion of the differences between the sulfathiazole polymorphs in terms of their molecular arrangements (PDF)

\section{AUTHOR INFORMATION}

\section{Corresponding Authors}

*E-mail: g.williams@ucl.ac.uk. Phone: +44 (0)207 7535868. Fax: +44(0)207 7535942 .

*E-mail: s.gaisford@ucl.ac.uk. Phone: +44 (0)207 7535863. Fax: +44(0)207 7535942 .

\section{Notes}

The authors declare no competing financial interest.

\section{ACKNOWLEDGMENTS}

The authors thank Diamond Light Source for access to Beamline I12 under Experiment EE10503-1, TA Instruments for the donation of a DSC instrument and technical advice, the EPSRC for funding under Grant Number EP/K03929/1, and UCL for the award of an Impact Studentship to A.C.

\section{REFERENCES}

(1) Scowen, I. J.; Telford, R. Simultaneous Thermal Techniques. In Principles of Thermal Analysis and Calorimetry; Gaisford, S., Kett, V., Haines, P. J., Eds.; Royal Society of Chemistry: Cambridge, U.K., 2016; pp 214-231.

(2) Mirabella, F. M. Appl. Spectrosc. 1986, 40 (3), 417-420.

(3) de Bakker, C. J.; St. John, N. A.; George, G. A. Polym. Prepr. 1992, 33 (1), 374-375.

(4) Buanz, A. B. M.; Telford, R.; Scowen, I. J.; Gaisford, S. CrystEngComm 2013, 15 (6), 1031.

(5) Sprunt, J. C.; Jayasooriya, A.; Wilson, R. H. Phys. Chem. Chem. Phys. 2000, 2 (19), 4299-4305.

(6) Huang, J.; Dali, M. J. Pharm. Biomed. Anal. 2013, 86, 92-99.

(7) Ali, H. R. H.; Edwards, H. G. M.; Scowen, I. J. J. Raman Spectrosc. 2009, 40 (8), 887-892.

(8) Hafsia, K. Ben; Ponçot, M.; Chapron, D.; Royaud, I.; Dahoun, A.; Bourson, P. J. Polym. Res. 2016, 23 (5), 93.

(9) Kuo, P. C.; Lo, C. T.; Chen, C. Y. Polymer 2013, 54 (24), 66546662.

(10) de Villiers, M. M.; Terblanche, R. J.; Liebenberg, W.; Swanepoel, E.; Dekker, T. G.; Song, M. J. Pharm. Biomed. Anal. 2005, 38 (3), 435-441.

(11) Karjalainen, M.; Airaksinen, S.; Rantanen, J.; Aaltonen, J.; Yliruusi, J. J. Pharm. Biomed. Anal. 2005, 39 (1-2), 27-32. 
(12) Walton, R. I.; O’Hare, D. Chem. Commun. 2000, 2283-2291.

(13) Buanz, A.; Prior, T. J.; Burley, J. C.; Raimi-Abraham, B. T.; Telford, R.; Hart, M.; Seaton, C. C.; Davies, P. J.; Scowen, I. J.; Gaisford, S.; Williams, G. R. Cryst. Growth Des. 2015, 15 (7), 32493256.

(14) Gilbert, E. P.; Nelson, A.; Sutton, D.; Terrill, N.; Martin, C.; Lal, J.; Lang, E. Mol. Cryst. Liq. Cryst. 2005, 440 (1), 93-105.

(15) Bayés-García, L.; Calvet, T.; Àngel Cuevas-Diarte, M.; Ueno, S.; Sato, K. CrystEngComm 2013, 15 (2), 302-314.

(16) Brubach, J. B.; Jannin, V.; Mahler, B.; Bourgaux, C.; Lessieur, P.; Roy, P.; Ollivon, M. Int. J. Pharm. 2007, 336 (2), 248-256.

(17) Drakopoulos, M.; Connolley, T.; Reinhard, C.; Atwood, R.; Magdysyuk, O.; Vo, N.; Hart, M.; Connor, L.; Humphreys, B.; Howell, G.; Davies, S.; Hill, T.; Wilkin, G.; Pedersen, U.; Foster, A.; De Maio, N.; Basham, M.; Yuan, F.; Wanelik, K. J. Synchrotron Radiat. 2015, 22 (3), 828-838

(18) Basham, M.; Filik, J.; Wharmby, M. T.; Chang, P. C. Y.; El Kassaby, B.; Gerring, M.; Aishima, J.; Levik, K.; Pulford, B. C. A.; Sikharulidze, I.; Sneddon, D.; Webber, M.; Dhesi, S. S.; Maccherozzi, F.; Svensson, O.; Brockhauser, S.; Náray, G.; Ashton, A. W. J. Synchrotron Radiat. 2015, 22 (3), 853-858.

(19) Larson, A. C.; Von Dreele, R. B. General Structure Analysis System (GSAS); Los Alamos National Laboratory Report LAUR 86$748,2004$.

(20) Coelho, A. A.; Evans, J.; Evans, I.; Kern, A.; Parsons, S. Powder Diffr. 2011, 26 (S1), S22-S25.

(21) Munroe, Á.; Rasmuson, Å. C.; Hodnett, B. K.; Croker, D. M. Cryst. Growth Des. 2012, 12 (6), 2825-2835.

(22) Anwar, J.; Tarling, S. E.; Barnes, P. J. Pharm. Sci. 1989, 78 (4), $337-342$.

(23) Giron, D. Pharm. Sci. Technol. Today 1998, 1 (5), 191-199.

(24) Giron, D. J. Therm. Anal. Calorim. 1999, 56 (3), 1285-1304.

(25) Zeitler, J. A.; Newnham, D. A.; Taday, P. F.; Threlfall, T. L.; Lancaster, R. W.; Berg, R. W.; Strachan, C. J.; Pepper, M.; Gordon, K. C.; Rades, T. J. Pharm. Sci. 2006, 95 (11), 2486-2498.

(26) Espeau, P.; Négrier, P.; Corvis, Y. Cryst. Growth Des. 2013, 13 (2), 723-730.

(27) Bhattacharya, S.; Saraswatula, V. G.; Saha, B. K. Cryst. Growth Des. 2013, 13 (8), 3651-3656.

(28) Grip, J.; Samuelsen, E. J. Phys. Scr. 1984, 29 (6), 556-560. 